\title{
Die Unterkühlbarkeit wässeriger Lösungen und ihre Bedeutung für die atmosphärische Eisbildung
}

\author{
Von WaLter RaU \\ Aus dem Max-Planck-Institut für Physik der Stratosphäre, Weißenau \\ (Z. Naturforschg. 9a, 939-944 [1954]; eingegangen am 16. August 1954)
}

\begin{abstract}
An NaCl-Lösungen verschiedener Konzentration wird das beim reinen Wasser gültige Gefrierkernspektrum und dieselbe Beeinflussung durch Impfen mit Kernen nachgewiesen. Es tritt aber eine Verschiebung um die jeweilige Gefrierpunktserniedrigung zu tieferen Temperaturen auf. Andere Salze und organische Stoffe haben dieselbe Wirkung.

Das zur Gefrierpunktserniedrigung durch Druck und zur Verschiebung des Dichtemaximums des Wassers durch Druck bzw. gelöste Stoffe analoge Verhalten führt wieder zu dem Schluß, daß das Gefrierkernspektrum durch Struktureigenschaften des Wassers verursacht wird.

Die Ergebnisse liefern eine passende Erklärung für das starke Anwachsen der atmosphärischen Eiskeimbildung bei Temperaturen zwischen -30 und $-40^{\circ} \mathrm{C}$.
\end{abstract}

$\mathrm{D}$ ie neueren Erkenntnisse über die Eigenschaften der natürlichen Aerosolteilchen ${ }^{1}$ machen es wahrscheinlich, daß die atmosphärische Eisbildung zum großen Teil in Lösungströpfchen vor sich geht. Damit erhebt sich die Frage nach deren Unterkühlbarkeit.

Gegenüber reinem Wasser tritt hier erschwerend auf, daß aus Lösungen je nach Konzentration verschiedene Phasen ausfallen. Das ist für das meteorologisch besonders interessierende Beispiel $\mathrm{NaCl}$-Wasser aus dem bekannten Schmelzpunktsdiagramm (Abb. 1) zu ersehen. Kochsalzlösungen sind oberhalb des Kurvenzuges ABCD beständig.

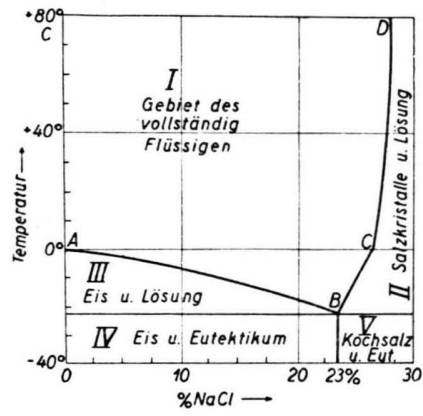

Abb. 1. Schmelzpunktsdiagramm NaCl-Wasser (nach Riesenfeld, Anorg. Chemie, Wien 1939).

Ist die Konzentration geringer als am eutektischen Punkt B, so fällt bei Kühlung zuerst Eis aus, die restliche Lösung wird konzentrierter und erstarrt schließlich eutektisch in B. Entsprechend fällt

1 Z. B. Ch. Junge, Ann. Met. 1952, Beiheft; H. Dessens, Ann. Géophys. 3, 1, 68 [1947]; D. Kuroiwa, J. Fac. Sci. Hokkaido Univ., [1953]. bei Konzentrationen oberhalb $\mathrm{B}$ zuerst $\mathrm{NaCl}$ aus, der Lösungsrest wird verdünnt und erstarrt wieder in B. Alle Konzentrationen besitzen somit ein Erstarrungsintervall, nur die eutektische Lösung hat den definierten Erstarrungspunkt B.

Diese Gleichgewichtsbedingungen gelten für bereits bestehende Phasen. Müssen dieselben neu gebildet werden, so ist entsprechend der Unterkühlbarkeit desWassers mit Abweichungen zu rechnen, deren Untersuchung weiteren Einblick in die Wirkungsweise der Gefrierkerne bzw. die Ursachen des Gefrierkernspektrums verspricht. Es ist besonders zwischen folgenden Möglichkeiten zu entscheiden :

1. Der von $\mathrm{Wall}{ }^{2}$ bei der Diskussion der Lösungskerne gemachte Ansatz, daß sich die Gefrierpunktserniedrigung zur Unterkühlung des Wassers addiert, setzt eine definierte Unterkühlung des Wassers voraus, die in Wirklichkeit nur in Form des Gefrierkernspektrums ${ }^{3}$ existiert. Das hat zur Folge, daß das Gefrierkernspektrum auch in Lösungen vorhanden sein müßte, nur eben um die Gefrierpunktserniedrigung verschoben. Daraus würde erneut folgen, daß das Gefrierkernspektrum durch Struktureigenschaften des Wassers bedingt ist $^{4}$.

2. Wären nämlich die spezifischen Kerneigenschaften (Isomorphie u. ä.) allein für das Ansprechen der Gefrierkerne maßgebend, so sollte die Gefrierpunktserniedrigung keinen Einfluß auf die

\footnotetext{
2 E. Wall, Met. Z. 60, 94 [1943].

${ }^{3}$ W. R au, Geofis. Pura Appl. 26, 75 [1953].

${ }^{4}$ W. Rau, Z. Naturforschg. 8a, 197 [1953].
} 
Eiskeimbildung haben, solange zuerst Eis ausfallen muß, d. h. für Konzentrationen unterhalb der eutektischen. Es bliebe also das normale Gefrierkernspektrum erhalten, nur der im Temperaturbereich der Gefrierpunktserniedrigung liegende Teil wäre abgeschnitten.

3. Falls Lösungen, wie häufig behauptet wird, überhaupt nicht unterkühlbar sind, darf gar kein Spektrum auftreten.

Die experimentelle Untersuchung geschah wieder nach den beim reinen Wasser angewandten Verfahren.

\section{NaCl-Wassertropfen a uf Metall}

Die zu untersuchenden Lösungen wurden aus wasserfreiem $\mathrm{NaCl}$ und doppelt destilliertem Wasser hergestellt. Davon wurden Serien von Tropfen auf hochglanzpolierten Chromflächen in der früher beschriebenen geschlossenen Kältekammer gekühlt und besonders darauf geachtet, daß Tropfenunterlage und Kammerluft dieselbe Temperatur hatten. Diese wurde mit Thermoelementen gemessen. Wegen des statistischen Volumeneffekts ${ }^{4}$ wurden wieder einheitlich Tropfen von $0,6 \mathrm{~cm}^{3}$ verwendet.

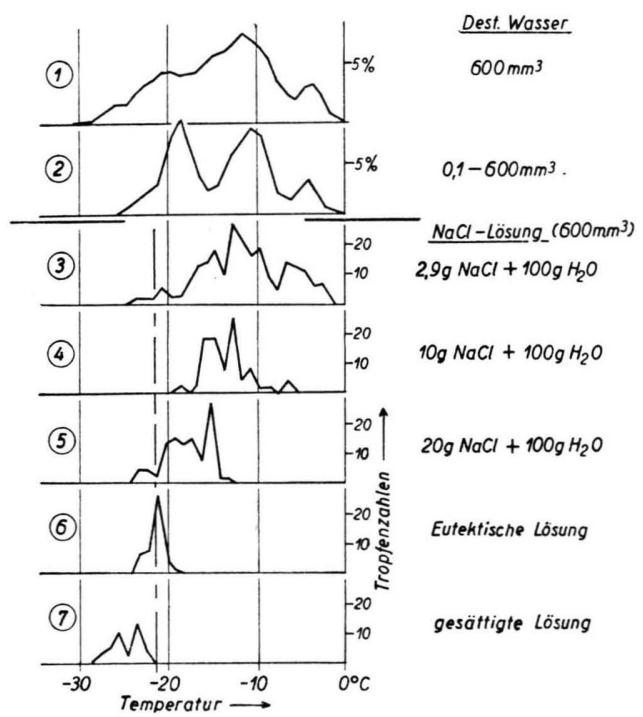

Abb. 2. Häufigkeitsverteilung der Erstarrungstemperatur in Tropfen. $1-2$ dest. Wasser; 3-7 NaClLösungen.

Lösungstropfen gleicher Konzentration erstarrten bei verschiedenen Temperaturen; Lösungen sind somit unterkühlbar. In Abb. 2 sind daher wieder Häufigkeitsverteilungen der Erstarrungstemperaturen wiedergegeben und zwar für verschiedene Konzentrationen, Kurven 3 bis 7. Zum Vergleich zeigen 1 und 2 die Verteilungen bei destilliertem
Wasser für gleich große bzw. ein Gemisch von verschieden großen Tropfen. Trägt man diese Erstarrungsintervalle in das Schmelzpunktsdiagramm ein (Abb. 3,a), so sieht man, daß Unterkühlungen tatsächlich vorkommen, solange zuerst das Eis ausfällt. Diese Unterkühlungen unterschreiten aber nicht den eutektischen Punkt. Lösungen der eutektischen Konzentration erweisen sich als nicht unterkühlbar. Die Kurve der ,,mittleren Unterkühlbarkeit" (= arithmetisches Mittel der Gefriertemperaturen) geht von $-11,8^{\circ} \mathrm{C}$ für das reine Wasser zum eutektischen Punkt B. Die höher gesättigten Lösungen erstarren etwas später.

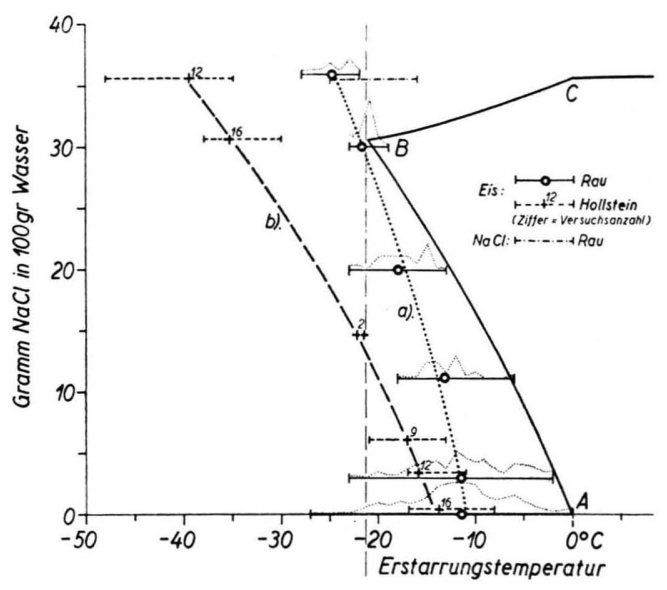

Abb. 3. Mittlere Erstarrungstemperatur von NaClLösungen in Abhängigkeit von der Konzentration. a) Tropfen; b) in Glasampullen nach Hollstein ${ }^{5}$.

In der ,Dunkelfeldbeleuchtung“ der Tropfen auf den Spiegeln sind die kristallinen Abscheidungen leicht zu erkennen und zu unterscheiden. Die Eisgebilde sind fein gefiederte hexagonale Diagonalsterne oder Teile von solchen und wachsen mit zunehmender Konzentration immer langsamer. Der Rest bleibt zunächst klar, geht aber bei weiterer Kühlung in das intensiv weiße Eutektikum über. Beginnt die Kristallisation mit dem NaCl, so geschieht das in Platten (monoklin, $\mathrm{NaCl}+2 \mathrm{H}_{2} \mathrm{O}$ ), die bei weiterer Kühlung Eutektikum ansetzen. So übersichtlich sind die Verhältnisse aber nur bei sehr langsamer Kühlung, bei rascher Kühlung wird der eutektische Punkt überschritten. Die NaClLösungen lassen sich übersättigen, in diesen geringen Mengen offenbar besonders leicht. Deshalb erstarren auch die hochkonzentrierten Lösungen in Abb. 2 erst so spät. Diese Übersättigung entspricht der Unterkühlbarkeit des reinen Wassers. Sie führt bei Temperaturen oberhalb des Nullpunkts zu der von Dessens und Jung ${ }^{1}$ an natürlichen und künstlichen Aerosolen beobachteten Hysteresis: Bei $77 \%$ rel. Feuchtigkeit gesättigte NaCl-Lösungströpfchen kristallisieren erst bei 30 bis $45 \%$ rel. Feuchte aus, lösen sich aber umgekehrt erst bei Sättigungsfeuchtigkeit wieder. 


\section{NaCl-Lösungen in Glasampullen}

Unser Ergebnis an NaCl-Lösungstropfen stimmt mit den Beobachtungen von $\mathrm{Holls}$ tein ${ }^{5}$ an Kochsalzlösungen in Glasröhren (Abb. 3, b) nicht überein, welche den obigen Fall 1 beweisen würden. Da jedoch die Zahl ihrer Messungen gering ist und nur die Werte bei höchsten Konzentrationen sicher abweichen, während die Werte bei niedrigen Konzentrationen mit unseren Ergebnissen noch verträglich wären, haben wir zur Klärung dieses in seinen Folgerungen wichtigen Widerspruchs unsere Kochsalzlösungen ebenfalls in Glasröhrchen eingeschmolzen untersucht ${ }^{6}$. Bei reinem Wasser hatte dieser Wechsel der Untersuchungsmethode keinen Einfluß, bei den Salzlösungen trifft das nicht mehr zu, Abb. 4. Wohl ergibt sich wieder eine Häufig-

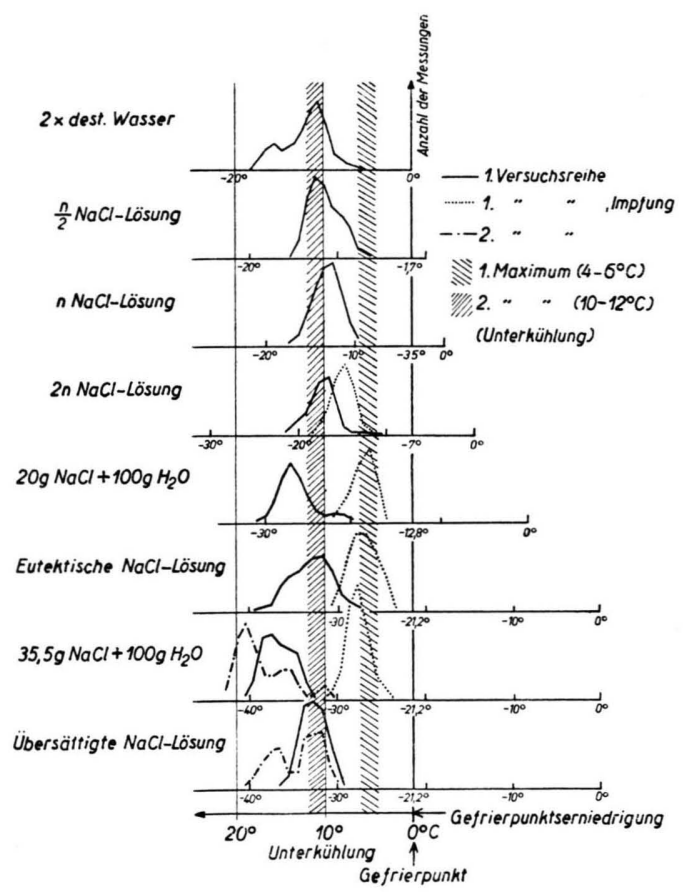

Abb. 4. Gefrierkernspektrum für NaCl-Lösungen in Glasampullen.

keitsverteilung der Gefriertemperaturen und sogar ein ausgeprägtes Maximum, dieses verschiebt sich aber tatsächlich wie bei Hollstein mit zunehmender Konzentration zu tieferen Temperaturen.

Wenn man daher als Abszisse die Unterkühlung (unter den erniedrigten Gefrierpunkt) und nicht

5 E. Hollstein, nach H.Weickmann, Ber. Dtsch. Wetterd. US-Zone Nr. 6, Bad Kissingen (1949).

6 Die Glasampullen wurden mit Hilfe eines HöplerThermostaten gekühlt. die Gefriertemperatur selbst wählt, die bei den einzelnen Verteilungen angeschrieben ist, so kommen die Maxima für verschiedene Konzentrationen untereinander, Abb.4.Im Schmelzpunktsdiagramm (Abb. 5) liegen die Werte für die mittlere Unterkühlbarkeit wie bei Hollstein parallel zur Löslichkeitskurve AB. Die gegenüber Hollstein durch-

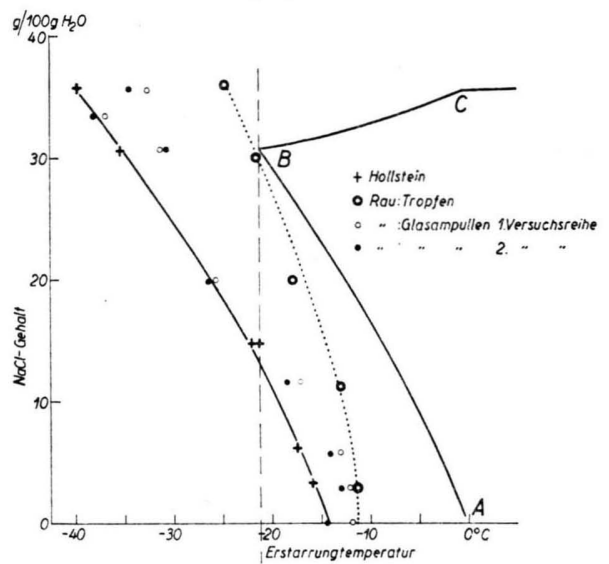

Abb. 5. Mittlere Erstarrungstemperaturen von NaClLösungen in Abhängigkeit von der Konzentration.

schnittlich $2^{0}$ höheren Mitteltemperaturen können durch unsere größere Statistik bzw. genauere Temperaturbestimmung verursacht sein. Dieser durch mehrfache Wiederholung der Versuche ${ }^{7}$ bestätigte Unterschied gegenüber den Tropfenuntersuchungen muß von der Untersuchungsmethode herrühren, und zwar daher, daß die Salzwassertropfen auf den Spiegeln abdampfen können, in den zugeschmolzenen Röhrchen nicht. Deshalb tritt auf den Spiegeln bei hoher Konzentration keine Übersättigung ein und es bilden sich auch bei geringeren Konzentrationen schon impfende Randablagerungen (Kerne bzw. Salz) und rufen wie beim reinen Wasser das erste Maximum des Gefrierkernspektrums um $-4^{0} \mathrm{C}$, bei Lösungen entsprechend später, aber auch schon in der Nähe des erniedrigten Gefrierpunkts hervor. Mit zunehmendem Salzgehalt nimmt die Abdampfgeschwindigkeit zu, entsprechend auch die Impfwirkung. Der an Tropfen gefundene Verlauf der mittleren Unterkühlbarkeit in Abb. 3 stellt somit nur einen auf diese Weise erzwungenen Übergang vom zweiten ins erste Maximum dar.

\footnotetext{
7 Serie 1 und 2 wurden von verschiedenen Beobachtern gemessen.
} 
Die Richtigkeit dieser Deutung folgt daraus, daß es gelingt, auch in geschlossenen Röhrchen das erste Maximum hervorzurufen. Man muß dazu nur den oberen Teil der Gläschen aus dem Kühlbad herausragen lassen und (z. B. durch einen warmen Luftstrom) erwärmen. So sind die punktierten Verteilungen der Abb. 4 gewonnen. Zum Teil ist direkt zu erkennen, wie die Eisbildung vom Meniskus ausgeht. Oft ist dies nicht möglich, wie überhaupt die Eisbildung in den Gläschen schlechter zu verfolgen ist und Eiskeime längst nicht so eindeutig zu lokalisieren sind wie auf den Metallflächen.

Die Beobachtungen an Kochsalzlösungen sind dahin zusammenzufassen, daß bei ihnen das Gefrierkernspektrum in der vom reinen Wasser her bekannten Weise wieder auftritt, und zwar bei der untersuchten Tropfengröße vorwiegend das zweite Maximum. Die Gefrierpunktserniedrigung addiert sich tatsächlich zur Unterkühlung. Nur bei den höchsten Konzentrationen (Abb. 5) ist evtl. eine Abweichung $\mathrm{zu}$ erkennen. Besonders wichtig ist aber, daß die Lösungstropfen leicht verdampfen, und dann tritt wieder in gewohnter Weise das erste Maximum (durch Impfung) auf.

\section{Weitere wässerige Lösungen}

Nach dem Raoultschen Gesetz hängt die Gefrierpunktserniedrigung bei einem bestimmten Lösungsmittel nur von der Anzahl der gelösten Moleküle und nicht von deren chemischer Natur ab. Dasselbe muß für die Verschiebung des Gefrierkernspektrums gelten. Dies wurde durch Untersuchungen an $\mathrm{CaCl}_{2}$, Traubenzucker, Rohrzucker, Glycerin und Äthylalkohol nachgewiesen, Abb. 6 . Als Abszisse ist hier wieder die Unterkühlung eingetragen, bei $0^{0}$ liegen also die erniedrigten Gefrierpunkte übereinander. Man sieht, daß die Maxima der Verteilungen dann auch wieder übereinander im Bereich des 2. Maximums angeordnet sind. Dabei ist $\mathrm{CaCl}_{2}$ ein dem $\mathrm{NaCl}$ ähnliches Salz, das den Gefrierpunkt stark erniedrigt und auch dissoziiert und somit das Raoultsche Gesetz nicht streng erfüllt, während die Zucker als organische

\footnotetext{
${ }^{8}$ Für Glycerin-Wasser ist die Gefrierpunktserniedrigung nach dem Raoultschen Gesetz berechnet, weil die Angaben in den verwendeten Tabellenwerken (H ütte I, Taschenbuch d. Ingenieurs; J. D' Ans und E. Lax, Taschenbuch für Chemiker und Physiker, Berlin, Göttingen, Heidelberg 1949) voneinander abweichen. Der berechnete Wert liegt für die geringere
}

Stoffe das Raoultsche Gesetz streng befolgen, wegen ihres hohen Molekulargewichts aber trotz Zugabe großer Mengen nur eine geringe Gefrierpunktserniedrigung hervorrufen. Äthylalkohol ist als Beispiel für einen Stoff gewählt, der aus der Lösung nicht auskristallisieren kann. Die Glycerin-Wasser-Gemische kristallisieren ausnahmsweise im ersten Maximum ${ }^{8}$. Die Abweichungen sind erstaunlich gering.

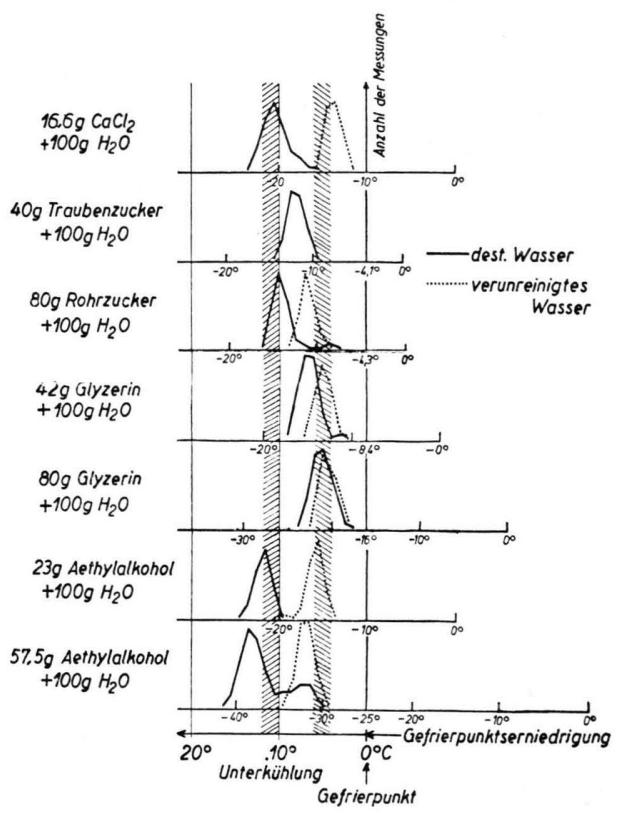

Abb. 6. Gefrierkernspektrum in weiteren wässerigen Lösungen.

4. Die Einwirkung von im Wasser enthaltenen festen Verunreinigungen (Kernen)

Werden die Lösungen mit Wasser angesetzt, das bezüglich Gefrierkernen als verunreinigt anzusprechen ist und selbst vorwiegend im 1. Maximum erstarrt, so tritt beim Erstarren wieder das 1. Maximum auf, allerdings verschoben, - Abb. 6, punktierte Verteilungen. Die früh ansprechenden Kerne behalten also ihre Wirksamkeit, die nach den bisherigen Untersuchungen darin besteht, daß sie unterkühltes Wasser bzw. hier unterkühlte Lösungen impfen und so die Eisbildungen knapp unter-

Konzentration zwischen beiden Tabellenwerten. Bei der höheren Konzentration ist in den Tabellen $25^{\circ}$ Gefrierpunktserniedrigung angegeben. Das kann aber nicht zutreffen, denn die beobachteten Erstarrungen können nicht oberhalb des erniedrigten Gefrierpunktes liegen. 
halb des Gefrierpunktes oder erniedrigten Gefrierpunktes hervorrufen. Darauf muß auch das frühe Erstarren der Glycerinlösung zurückgehen. Wegen weiterer Einzelheiten über diese Impfung und ihre Erklärung verweisen wir auf frühere Arbeiten ${ }^{3}$.

$\mathrm{Zu}$ bemerken ist noch, daß auch hier alle ausfallenden Eisgebilde hexagonale Kristallstruktur ${ }^{9}$ haben und in den Lösungen wieder bei zunehmender Konzentration immer langsamer wachsen.

\section{Volumeneffekt}

Wie bereits erwähnt, tritt hier immer nur das 1. und 2. Maximum des Gefrierkernspektrums auf. Da die Tropfen $0,6 \mathrm{~cm}^{3}$ groß waren, entspricht dies genau den Beobachtungen am reinen Wasser. Das 3. Maximum bei $-19^{\circ} \mathrm{C}$ sollte bei entsprechender Alterung oder kleineren Tropfenvolumen herauskommen. In unseren Versuchen kam es nur in Ampullen, und zwar bei den stark gesättigten NaCl-Lösungen vor (Abb. 4). Es auch durch kleinere Tröpfchen zu verifizieren, gelang nicht gut. Kleine, übersättigte NaCl-Wassertröpfchen erstarrten zwar vorwiegend $a b-40^{\circ} \mathrm{C}$, also im verschobenen 3. Maximum ${ }^{10}$. Die Versuche sind aber nicht sehr eindeutig. Denn solche Tröpfchen dampfen teilweise rasch ab und erstarren dabei häufig früh (durch Impfung), teilweise sinkt aber auch ihre Konzentration durch hinzukondensiertes Wasser, was gerade bei kleinen Tropfen viel ausmachen kann.

\section{Folgerungen}

Die Untersuchungen an wässerigen Lösungen ergänzen somit die am reinen Wasser gewonnenen Vorstellungen. Die Kristallisation setzt an Kernen ein, und ihre Einzelheiten sind nicht ohne diese zu verstehen, die Wirkung der Kerne ist aber nicht an eine spezifische absolute Temperatur geknüpft. Die Bindung an bestimmte Temperaturbereiche erfolgt durch das Wasser selbst im Sinne der Deutung des Gefrierkernspektrums durch Modifikationsänderungen des Wassers. Das Gefrierkernspektrum gilt also nicht nur ganz allgemein bei

\footnotetext{
${ }^{9}$ Auch die hohe Alkoholkonzentration erzwingt noch nicht den Übergang zu der von uns bei $-72^{\circ} \mathrm{C}$ beobachteten kubischen Eismodifikation. Der Einwand von Cwilong (J. Glaciol. 1, 53 [1947]) bzw. Palmer und Brewer (Proc. Phys. Soc., Lond. B 64, 765 [1951]), daß Spuren von Alkohol für diese reguläre (kubische) Modifikation verantwortlich seien, ist damit hinfällig.
}

„,reinem“"Wasser, sondern auch bei Lösungen. Mit der nachgewiesenen Kopplung an den verschieden stark erniedrigten Gefrierpunkt zeigt es dasselbe Verhalten wie das Dichtemaximum des Wassers, das im reinen Wasser bei $+4^{\circ} \mathrm{C}$ liegt, in Lösungen aber zu tieferen Temperaturen rückt. Druckerhöhung hat dieselbe Wirkung, und zwar sowohl beim Dichtemaximum ${ }^{11}$ als auch beim Gefrierpunkt. Ob das auch beim Gefrierkernspektrum zutrifft, wäre noch zu prüfen.

Das Dichtemaximum kommt aber bekanntlich dadurch zustande, daß sich die Zusammensetzung des Wassers aus verschiedenen Molekülarten mit der Temperatur ändert. Dasselbe tritt also auch bei Druckänderungen oder bei Anwesenheit von gelösten Molekülen ein. Strukturänderungen des Wassers dürfen daher auch für die Verschiebung des Gefrierkernspektrums und damit auch für dieses selbst verantwortlich gemacht werden ${ }^{12}$. Im Grunde ändert sich immer der Dampfdruck des Wassers und das hat die Gefrierpunktserniedrigung (bzw. die hier nicht interessierende Siedepunktserhöhung) zur Folge. In diesem Zusammenhang darf an unseren Hinweis ${ }^{4}$, daß das 2. Maximum gerade bei $-12^{\circ} \mathrm{C}$ liegt, wo der Dampfdruckunterschied über Wasser und über Eis am größten ist, erinnert werden.

Die Anwendung dieser Ergebnisse auf die atmosphärische Eisbildung ist damit gegeben, daß die Lösungskerne als Kondensationskerne die ersten Ablagerungsstellen des Wasserdampfes, d. h. also die ersten größeren Tröpfchen sind. In ihnen ist die Eisbildung nicht um definierte Beträge verschoben, sondern je nach ihrer Konzentration, die sich (zusammen mit ihrer Größe und ihrem Dampfdruck) der relativen Feuchte ihrer Umgebung anpaßt, was zu der bekannten Abhängigkeit der Sichtweite in der Atmosphäre von der Feuchtigkeit führt.

Im abdampfenden oder abgedampften Zustand — ohne Berücksichtigung der von J ung e ${ }^{1}$ ermit. telten Hysterese ist das bei $\mathrm{NaCl}$ schon ab $75 \%$ rel. Feuchtigkeit der Fall - stellen solche Kerne gute Impfkerne dar ${ }^{13}$. Auf die daraus resultierende Be-

${ }^{10}$ Das ist auch von C. Larfargue (C. R. Acad. Sci., Paris 230, 1194 [1950]) beobachtet worden.

${ }_{11}$ P. W. Bridgman, Z. anorg. Chem. 77, 377 [1912].

${ }^{12}$ W. R a u, Z. Naturforschg. 5a, 667 [1950].

13 Daß nach Bigg (siehe B. J. Mason, Arch. Met. Geophys. Bioklimatolog. 6, 1 [1953]) gewisse Ionen eine (geringe) Erhöhung der Erstarrungstemperatur des reinen Wassers bringen sollen, ist in dieser sekun- 
deutung von Inversionen für die Eisbildung wurde schon hingewiesen.

In feuchtem Zustand verschieben die Mischkerne als Lösungen das Gefrierkernspektrum des reinen Wassers bzw. können sie bei entsprechend tiefen Temperaturen selbst erstarren und dann direkt als Eiskerne zur Wirkung kommen. Mit der maximal möglichen Gefrierpunktserniedrigung von $21,5^{\circ} \mathrm{C}$ durch $\mathrm{NaCl}$ (eutektischer Punkt) kämen die drei Maxima des Gefrierkernspektrums auf $-25^{\circ} \mathrm{C}$, $-32^{\circ} \mathrm{C}$ und $-40,5^{\circ} \mathrm{C}$ zu liegen. Dazu paßt, daß schon Findeisen ${ }^{14}$ eine starke, von uns inzwischen bestätigte $^{15}$ Zunahme der Eisbildung (seine Kerne 2. Art) bei $-33^{\circ} \mathrm{C}$ angibt, daß Schaefer ${ }^{16}$ u. a. diese Zunahme bei -39 bzw. $-41^{\circ} \mathrm{C}$ beobachten und daß $\mathrm{C}$ wilong ${ }^{17}$ seine kritischen Eisbildungstemperaturen bei $-32^{\circ} \mathrm{C}$ und $-41^{\circ} \mathrm{C}$ ansetzt. Die gute Übereinstimmung wird dadurch beeinträchtigt, daß diese kritischen Temperaturen nicht einheitlich gefunden und zum Teil sogar überhaupt bestritten worden sind und daß bei $-40^{\circ} \mathrm{C}$ vielfach schon die homogene, d. h. ohne Mitwirkung

dären Größenordnung denkbar, durch den Vergleich mit einer festen Erstarrungstemperatur des reinen Wassers von $-23^{\circ} \mathrm{C}$ aber nicht zu erweisen. Hier muß die den Gefrierkernen gerechte spektrale Verteilung berücksichtigt werden und die Tatsache, daß bei Impfung wesentlich stärkere Erhöhungen auftreten, auch ohne Ionen. Da seine Tropfen in die Grenzschicht zwischen verschiedenen organischen Stoffen eingebettet sind, sind die Impfung begünstigende Vorgänge denkbar, etwa durch chemische Reaktion oder indem sich hydrophobe Stoffe in die Grenzschicht drängen von Kernen stattfindende Eiskeimbildung angesetzt worden ist. Nachdem sich aber zeigen läßt ${ }^{18}$, daß die Keimbildung bei $-40^{\circ} \mathrm{C}$ wohl sehr stark, aber noch nicht homogen ist, muß hier eine beachtliche Kernzahl wirksam werden. Das deutet wieder auf Lösungskerne hin, zumal gerade diese bei der raschen Kondensation in sehr schnell arbeitenden Expansionskammern oder in Taubeschlägen bei tiefen Temperaturen u. U. auch leicht unwirksam werden können, was die bei Temperaturen unter $-40^{\circ} \mathrm{C}$ beobachteten Unterkühlungen erklären würde. Als weiterer Hinweis in dieser Richtung ist zu werten, daß die Eisbildung gerade über See früher einsetzt als über Land.

Auf jeden Fall bietet diese Erklärung den Vorteil, daß nicht ganz unbekannte hypothetische und sogar mit Eis isomorphe Kerne zur Deutung herangezogen werden, sondern nur die bei der Kondensation sowieso und sogar in erster Linie beteiligten Mischkerne, welche eine durch die vorgelegten Untersuchungen begründete Wirksamkeit als Gefrierkerne besitzen müssen.

(s. H. Freundlich, Kapillarchemie, Leipzig 1922, S. 215). Zu letzteren gehören gerade die bei ihm wirksamen Halogenide (AgJ, $\mathrm{HgJ}_{2}, \mathrm{PbJ}_{2}$ u. a.).

${ }^{14}$ W. Findeisen u. G. Schulz (1944), Ber. Deutsch. Wetterd. US-Zone Nr. 1 [1947].

${ }^{15}$ W. Rau, Met. Rundschau 7, 59 [1954].

${ }^{16}$ V. J. Schaefer, Bull. Amer. Met. Soc. 30, 142 [1949] u. a.

17 B. M. Cwilong, Proc. Roy. Soc. A 190, 137 [1947].

${ }^{18} \mathrm{~W}$. Rau, erscheint demnächst.

\title{
Über die aus Ultraschallversuchen und dielektrischen Messungen ermittelte Relaxationszeit und ihre Abhängigkeit von der Viskosität
}

\author{
Von Arno K. Schulz \\ Aus dem Physikalischen Institut der Universität Saarbrücken \\ (Z. Naturforschg. 9a, 944-950 [1954]; eingegangen am 26. Juli 1954)
}

\begin{abstract}
Von dielektrischen Messungen an flüssigem, glasigem und kristallinem Glycerin im Frequenzgebiet von 0 bis $10^{6} \mathrm{~Hz}$ ausgehend wurde unter Einbezug von Literaturwerten $\left(\boldsymbol{v}=10^{6}\right.$ bis $\left.2,2 \cdot 10^{8} \mathrm{~Hz}\right)$ die mittlere Relaxationszeit von unterkühltem Glycerin in $\mathrm{Ab}$ hängigkeit von der Temperatur über etwa 11 Zehnerpotenzen ermittelt und mit den entsprechenden Werten verglichen, die experimentell aus Ultraschallmessungen bzw. rechnerisch auf Grund einer Modellvorstellung aus der Viskosität und der adiabatischen Kompressibilität erhalten wurden.
\end{abstract}

$\mathrm{G}$ lycerin besitzt einen kristallinen Schmelzpunkt von $+18^{\circ} \mathrm{C}$. Im Gegensatz zu zahlreichen anderen Flüssigkeiten kristallisiert Glycerin beim Abkühlen unter den kristallinen Schmelzpunkt praktisch nie, sondern bleibt auch unterhalb $+18^{\circ} \mathrm{C}$ flüssig. Mit wachsender Unterkühlung steigt die Viskosität von etwa 10 Poise bei Zimmertemperatur auf ungefähr $10^{13}$ Poise im Gebiet der Trans- 